\title{
EFFECT OF ISOVECTOR-SCALAR MESON ON NEUTRON STAR MATTER IN STRONG MAGNETIC FIELDS
}

\author{
F.X. Wei*1, G.J. $\mathrm{Mao}^{2,3}$, C. M. Ko ${ }^{4}$, L.S. Kisslinger ${ }^{5}$, \\ H. Stöcker ${ }^{6}$, and W. Greiner ${ }^{6}$ \\ ${ }^{1}$ Institute of High Energy Physics, Chinese Academy of Sciences, \\ P.O.Box 918(4), Beijing 100049, China ${ }^{\dagger}$ \\ ${ }^{2}$ CCAST (World Laboratory), P.O.Box 8730, Beijing 100080, China \\ ${ }^{3}$ Department of Physics, Beihang University, Beijing 100083, China \\ ${ }^{4}$ Cyclotron Institute and Physics Department, Texas A-M University, \\ College Station, Texas 7r843-3366 \\ ${ }^{5}$ Department of Physics, Carnegie Mellon University, Pittsburgh PA 15213 \\ ${ }^{6}$ Frankfurt Institute for Advanced Studies, Johann Wolfgang Goethe-University, \\ Max-von-Laue-Str. 1, D-60438 Frankfurt am Main, Germany
}

\begin{abstract}
We study the effects of isovector-scalar meson $\delta$ on the equation of state (EOS) of neutron star matter in strong magnetic fields. The EOS of neutron-star matter and nucleon effective masses are calculated in the framework of Lagrangian field theory, which is solved within the mean-field approximation. From the numerical results one can find that the $\delta$-field leads to a remarkable splitting of proton and neutron effective masses. The strength of $\delta$-field decreases with the increasing of the magnetic field and is little at ultrastrong field. The proton effective mass is highly influenced by magnetic fields, while the effect of magnetic fields on the neutron effective mass is negligible. The EOS turns out to be stiffer at $B<10^{15} \mathrm{G}$ but becomes softer at stronger magnetic field after including the $\delta$-field. The AMM terms can affect the system merely at ultrastrong magnetic field $\left(B>10^{19} \mathrm{G}\right)$. In the range of $10^{15} \mathrm{G}-10^{18} \mathrm{G}$ the properties of neutron-star matter are found to be similar with those without magnetic fields.
\end{abstract}

PACS numbers: 26.60.+c, 21.65. $+\mathrm{f}$

\footnotetext{
*E-mail: weifx@ihep.ac.cn

${ }^{\dagger}$ mailing address
} 


\section{Introduction}

In the standard relativistic mean field (RMF) model $[1,2]$ of nuclear matter, the $\sigma$ , $\omega$ and $\rho$ meson are used in descriptions of nuclear interactions. The short range of the isovector-scalar meson $a_{0}(980)$ (the $\delta$ meson) exchange justifies neglecting its contribution to symmetric nuclear matter. However, for strongly isospin-asymmetric matter at high densities in neutron stars, the contribution of the $\delta$-field should be considered. Resent theoretical studies $[3,4,5,6,7]$ motivate the investigation of the effect of the isovectorscalar meson on the neutron-star matter. They have found [4] that the $\delta$-field leads to a large repulsion in dense neutron-rich matter and definite splitting of proton and neutron effective masses. The energy per particle of neutron matter becomes larger at high densities than the one with no $\delta$-field included and the proton fraction of $\beta$-stable matter increases[5]. Those properties play an important role in description of the structure and stability conditions of neutron stars. A splitting of proton and neutron masses can affect the transport properties of dense matter [8]. As is well known, there are strong magnetic fields of $10^{14} \mathrm{G}[9]$ on the neutron star surface. The strength of magnetic fields in the interior of neutron stars can be up to $10^{18} G$ [10]. The neutron-star matter in strong magnetic fields without the isovector-scalar field has been studied, which give interesting and novelty results $[11,12,13]$. It is our main aim to investigate the $\delta$-field influence on the properties of the neutron-star matter in the presence of magnetic fields.

Theoretical studies about the effects of very strong magnetic fields on the EOS of neutron-star matter indicated[11] that the softening of the EOS caused by Landau quantization was overwhelmed by stiffening due to the incorporation of the anomalous magnetic moments of nucleons. At high baryon densities, muons can be produced in the chargeneutral, beta-equilibrated matter with respect to the channel of $e^{-} \longleftrightarrow \mu^{-}+\nu_{e}+\bar{\nu}_{\mu}$ , as soon as the chemical potential of electrons $\mu_{e}$ reaches a value equaling to the muon rest mass. In cold neutron stars neutrinos and photons already escape and the chemical potentials of those can be set as zero. Consequently, we get $\mu_{e}=\mu_{\mu}\left(\mu_{\mu}\right.$ is the chemical 
potential of muons). As the baryon density increases, the densities of muons and electrons become comparable with that of nucleons. The inclusion of the anomalous magnetic moments of leptons thus makes sense. In this paper we will study the effects of the AMM of nucleons and leptons in a dense neutron-star matter including the $\delta$-field effect.

In the following section, the Lagrangian field theory of interacting nucleons and mesons including magnetic fields will be introduced. The numerical results are given in section 3. We separate the cases with and without the inclusion of the AMM effects. The modification of proton and neutron effective masses in the dense matter with strong magnetic fields will be discussed in detail. The energy per particle and EOS will be studied too. In Section 4, we summarize our results and prospect for the possible descriptions of additional components such as hyperons and quarks.

\section{Formalism}

The application of Lagrangian field theory to the study of neutron stars was first carried out by Glendenning[14]. We consider a neutron-star matter consisting of neutrons, protons, electrons and muons in the presence of a uniform magnetic field B along the zaxis. The Lagrangian density can be written as

$$
\begin{aligned}
\mathcal{L}= & \bar{\psi}_{b}\left[i \gamma_{\mu} \partial^{\mu}-q_{b} \frac{1+\tau_{0}}{2} \gamma_{\mu} A^{\mu}-\frac{1}{4} \kappa_{b} \mu_{N} \sigma_{\mu \nu} F^{\mu \nu}-M_{b}+g_{\sigma} \sigma+g_{\delta} \boldsymbol{\tau} \cdot \boldsymbol{\delta}-g_{\omega} \gamma_{\mu} \omega^{\mu}-g_{\rho} \gamma_{\mu} \boldsymbol{\tau} \cdot \mathbf{R}^{\mu}\right] \psi_{b} \\
& +\bar{\psi}_{l}\left[i \gamma_{\mu} \partial^{\mu}-q_{l} \gamma_{\mu} A^{\mu}-\frac{1}{4} \kappa_{l} \mu_{B} \sigma_{\mu \nu} F^{\mu \nu}-m_{l}\right] \psi_{l}+\frac{1}{2} \partial_{\mu} \sigma \partial^{\mu} \sigma-U(\sigma)-\frac{1}{2} m_{\sigma}^{2} \sigma^{2}+\frac{1}{2} \partial_{\mu} \boldsymbol{\delta} \partial^{\mu} \boldsymbol{\delta} \\
& -\frac{1}{2} m_{\delta}^{2} \boldsymbol{\delta}^{2}-\frac{1}{4} \omega_{\mu \nu} \omega^{\mu \nu}+\frac{1}{2} m_{\omega}^{2} \omega_{\mu} \omega^{\mu}-\frac{1}{4} \mathbf{R}_{\mu \nu} \cdot \mathbf{R}^{\mu \nu}+\frac{1}{2} m_{\rho}^{2} \mathbf{R}_{\mu} \mathbf{R}^{\mu}-\frac{1}{4} F_{\mu \nu} F^{\mu \nu}
\end{aligned}
$$

where $\psi_{b}$ and $\psi_{l}$ are the baryon $(\mathrm{b}=\mathrm{n}, \mathrm{p})$ and lepton $(\mathrm{l}=\mathrm{e}, \mu)$ fields; $\sigma, \omega_{\mu}, \mathbf{R}, \boldsymbol{\delta}$ represent the scalar meson, vector meson, isovector-vector meson and isovector-scalar meson field, which are exchanged for the description of nuclear interactions. $A^{\mu} \equiv(0,0, B x, 0)$ refers to a constant external magnetic field along the $z$-axis. The field tensors for the $\omega, \rho$ and magnetic field are given by $\omega_{\mu \nu}=\partial_{\mu} \omega_{\nu}-\partial_{\nu} \omega_{\mu}, \mathbf{R}_{\mu \nu}=\partial_{\mu} \mathbf{R}_{\nu}-\partial_{\nu} \mathbf{R}_{\mu}$ and $F_{\mu \nu}=\partial_{\mu} A_{\nu}-\partial_{\nu} A_{\mu}$ . $\mathrm{U}(\sigma)$ is the self-interaction part of the scalar field[15]: $U(\sigma)=\frac{1}{3} b \sigma^{3}+\frac{1}{4} c \sigma^{4}$. 
$M_{b}$ and $m_{l}$ are free baryon masses and lepton masses, and $m_{\sigma}, m_{\omega}, m_{\rho}, m_{\delta}$ are the masses of the $\sigma, \omega, \rho$ and $\delta$ meson, respectively. $\mu_{N}$ and $\mu_{B}$ are the nuclear magneton of nucleons and Bohr magneton of electrons; $\kappa_{p}=3.5856, \kappa_{n}=-3.8263, \kappa_{l}=\frac{\alpha_{l}}{\pi}$ and $\alpha_{l}=1159652188(4) \times 10^{12}(4 p p b), \alpha_{\mu}=11659203(8) \times 10^{-10}(0.7 p p m)[16,17,18]$ are the coefficients of AMMs for protons, neutrons, electrons and leptons, respectively. Then the anomalous magnetic moment can be defined by the coupling of the baryons and leptons to the electromagnetic field tensor with $\sigma_{\mu \nu}=\frac{i}{2}\left[\gamma_{\mu}, \gamma_{\nu}\right]$ and $\kappa_{i}(i=n, p, e, \mu)$ given above.

The field equations in a mean field approximation(MFA), in which the meson fields are replaced by their expectation values in a many-body ground state, are given by

$$
\begin{aligned}
& \left(i \gamma_{\mu} \partial^{\mu}-q_{b} \frac{1+\tau_{0}}{2} \gamma_{\mu} A^{\mu}-\frac{1}{4} \kappa_{b} \mu_{N} \sigma_{\mu \nu} F^{\mu \nu}-m_{b}^{*}-g_{\omega} \gamma_{\mu} \omega^{\mu}-g_{\rho} \gamma_{0} \tau_{3 b} R_{0}^{0}\right) \psi_{b}=0 \\
& \left(i \gamma_{\mu} \partial^{\mu}-q_{l} \gamma_{\mu} A^{\mu}-\frac{1}{4} \kappa_{b} \mu_{N} \sigma_{\mu \nu} F^{\mu \nu}-m_{l}\right) \psi_{l}=0 \\
& \quad m_{\sigma}^{2} \sigma+b \sigma^{2}+c \sigma^{3}=g_{\sigma} \rho_{s} \\
& \quad m_{\omega}^{2} \omega_{0}=g_{\omega} \rho_{b} \\
& \quad m_{\rho}^{2} R_{00}=g_{\rho}\left(\rho_{p}-\rho_{n}\right) \\
& \quad m_{\delta}^{2} \delta_{0}=g_{\delta}\left(\rho_{s}^{p}-\rho_{s}^{n}\right) .
\end{aligned}
$$

The energy-momentum tensor can be written as

$$
\begin{aligned}
T_{\mu \nu}= & i \bar{\psi}_{b, l} \gamma_{\mu} \partial_{\nu} \psi_{b, l}+g_{\mu \nu}\left[\frac{1}{2} m_{\sigma}^{2} \sigma^{2}+U(\sigma)+\frac{1}{2} m_{\delta}^{2} \boldsymbol{\delta}^{2}-\frac{1}{2} m_{\omega}^{2} \omega_{\lambda} \omega^{\lambda}\right. \\
& \left.-\frac{1}{2} m_{\rho}^{2} \mathbf{R}_{\lambda} \mathbf{R}^{\lambda}+\frac{B^{2}}{2}\right]+\partial_{\nu} A^{\lambda} F_{\lambda \mu} .
\end{aligned}
$$

Here $\rho_{b}=\rho_{p}+\rho_{n}$ is the baryon number density, and $\rho_{s}=\rho_{s}^{p}+\rho_{s}^{n}$ is the scalar number density. In the mean field approximation, the first two components of the isospin vector $\mathbf{R}_{\mu}$ and $\boldsymbol{\delta}$ vanish; i.e, $\left\langle\mathbf{R}^{\mu}\right\rangle=\left\langle R_{0}^{\mu}\right\rangle ;\langle\boldsymbol{\delta}\rangle=\left\langle\delta_{0}\right\rangle$. The effective baryon masses are thus expressed as

$$
\begin{aligned}
& m_{p}^{*}=M_{p}-g_{\sigma} \sigma-g_{\delta} \delta_{0}, \\
& m_{n}^{*}=M_{n}-g_{\sigma} \sigma+g_{\delta} \delta_{0} .
\end{aligned}
$$


In the presence of the AMM of nucleons and leptons, the energy spectra of particles can be expressed as

$$
\begin{aligned}
E_{\nu, s}^{p} & =\sqrt{k_{z}^{2}+\left(\sqrt{2 e B \nu+m_{p}^{* 2}}+s \Delta_{p}\right)^{2}}+g_{\omega} \omega_{0}+g_{\rho} R_{0,0}, \\
E_{s}^{n} & =\sqrt{k_{z}^{2}+\left(\sqrt{k_{x}^{2}+k_{y}^{2}+m_{n}^{* 2}}+s \Delta_{n}\right)^{2}}+g_{\omega} \omega_{0}-g_{\rho} R_{0,0}, \\
E_{\nu, s}^{l} & =\sqrt{k_{z}^{2}+\left(\sqrt{2 e B \nu+m_{l}^{2}}+s \Delta_{l}\right)^{2}},
\end{aligned}
$$

where $\Delta_{b}=-\frac{1}{2} \kappa_{b} \mu_{N} B$ and $\Delta_{l}=-\frac{1}{2} \kappa_{l} \mu_{B} B$.

The number densities of protons, neutrons and leptons read as

$$
\begin{aligned}
\rho_{p} & =\frac{e B}{2 \pi^{2}}\left[\sum_{\nu=0}^{\nu_{\max }} k_{f, \nu, 1}^{(p)}+\sum_{\nu=1}^{\nu_{\max }} k_{f, \nu,-1}^{(p)}\right], \\
\rho_{n} & =\frac{1}{2 \pi} \sum_{s}\left\{\frac{2}{3} k_{f, s}^{(n) 3}+s \Delta_{n}\left[\left(m_{n}^{*}+s \Delta_{n}\right) k_{f, s}^{(n)}+E_{f}^{(n) 2}\left(\arcsin \frac{m_{n}^{*}+s \Delta_{n}}{E_{f}^{(n)}}-\frac{\pi}{2}\right)\right]\right\},
\end{aligned}
$$

and

$$
\rho_{l}=\frac{e B}{2 \pi^{2}}\left[\sum_{\nu=0}^{\nu_{\max }} k_{f, \nu, 1}^{(l)}+\sum_{\nu=1}^{\nu_{\max }} k_{f, \nu,-1}^{(l)}\right]
$$

respectively. In the above expressions, $k_{f, \nu, s}^{(p)}$ and $k_{f, \nu, s}^{(l)}$ are the Fermi momenta of protons and leptons for the landau level $\nu$ and the spin index $s=-1,1 ; k_{f, s}^{(n)}$ is the Fermi momentum of neutrons. They are related to the Fermi energies as

$$
\begin{aligned}
k_{f, \nu, s}^{(p)} & =\sqrt{E_{f}^{(p) 2}-\left(\sqrt{m_{p}^{* 2}+2 e B \nu}+s \Delta_{p}\right)^{2}}, \\
k_{f, s}^{(n)} & =\sqrt{E_{f}^{(n)}-\left(m_{n}^{*}+s \Delta_{n}\right)^{2}}
\end{aligned}
$$

and

$$
k_{f, \nu, s}^{(l)}=\sqrt{E_{f}^{(l) 2}-\left(\sqrt{m_{l}^{2}+2 e B \nu}+s \Delta_{l}\right)^{2}} .
$$


The scalar number densities of nucleons have the form of

$$
\begin{aligned}
\rho_{s}^{p} & =\frac{e B m_{p}^{*}}{2 \pi^{2}}\left[\sum_{\nu=0}^{\nu_{\max }} \frac{\sqrt{m_{p}^{* 2}+2 e B \nu}+\Delta_{p}}{\sqrt{m_{p}^{* 2}+2 e B \nu}} \ln \left|\frac{k_{f, n, 1}^{(p)}+E_{f}^{(p)}}{\sqrt{m_{p}^{* 2}+2 e B \nu}+\Delta_{p}}\right|\right. \\
& \left.+\sum_{\nu=1}^{\nu_{\max }} \frac{\sqrt{m_{p}^{* 2}+2 e B \nu}-\Delta_{p}}{\sqrt{m_{p}^{* 2}+2 e B \nu}} \ln \left|\frac{k_{f, n,-1}^{(p)}+E_{f}^{(p)}}{\sqrt{m_{p}^{* 2}+2 e B \nu}-\Delta_{p}}\right|\right], \\
\rho_{s}^{n} & =\frac{m_{n}^{*}}{4 \pi^{2}} \sum_{s}\left[k_{f, s}^{(n)} E_{f}^{(n)}-\left(m_{n}^{*}+s \Delta_{n}\right)^{2} \ln \left|\frac{k_{f}^{(n)}+E_{f}^{(n)}}{m_{n}^{*}+s \Delta_{n}}\right|\right] .
\end{aligned}
$$

The energy densities of nucleons and leptons are given as

$$
\begin{aligned}
\varepsilon_{p}= & \frac{1}{4 \pi^{2}} \sum_{\nu=0}^{\nu_{\max }}\left[k_{f, \nu, 1}^{(p)} E_{f}^{(p)}+\left(\sqrt{m_{p}^{* 2}+2 e B \nu}+\Delta_{p}\right)^{2} \ln \left|\frac{k_{f, \nu, 1}^{(p)}+E_{f}^{(p)}}{\sqrt{m_{p}^{* 2}+2 e B \nu}+\Delta_{p}}\right|\right] \\
& +\frac{1}{4 \pi^{2}} \sum_{\nu=1}^{\nu_{\max }}\left[k_{f, \nu,-1}^{(p)} E_{f}^{(p)}+\left(\sqrt{m_{p}^{* 2}+2 e B \nu}-\Delta_{p}\right)^{2} \ln \left|\frac{k_{f, \nu,-1}^{(p)}+E_{f}^{(p)}}{\sqrt{m_{p}^{* 2}+2 e B \nu}-\Delta_{p}}\right|\right], \quad(22) \\
\varepsilon_{n}= & \frac{1}{4 \pi^{2}} \sum_{s}\left\{\frac{1}{2} k_{f, s}^{(n)} E_{f}^{(n) 3}+\frac{2}{3} s \Delta_{n} E_{f}^{(n) 3}\left(\arcsin \frac{m_{n}^{*}+s \Delta_{n}}{E_{f}^{(n)}}-\frac{\pi}{2}\right)+\left(\frac{s \Delta_{n}}{3}-\frac{m_{n}^{*}+s \Delta_{n}}{4}\right)\right. \\
& \left.\times\left[\left(m_{n}^{*}+s \Delta_{n}\right) k_{f, s}^{(n)} E_{f}^{(n)}+\left(m_{n}^{*}+s \Delta_{n}\right)^{3} \ln \left|\frac{k_{f}^{(n)}+E_{f}^{(n)}}{m_{n}^{*}+s \Delta_{n}}\right|\right]\right\} \\
\varepsilon_{l}= & \frac{1}{4 \pi^{2}} \sum_{\nu=0}^{\nu_{\max }}\left[k_{f, \nu, 1}^{(l)} E_{f}^{(l)}+\left(\sqrt{m_{l}^{2}+2 e B \nu}+\Delta_{l}\right)^{2} \ln \left|\frac{k_{f, \nu, 1}^{(l)}+E_{f}^{(l)}}{\sqrt{m_{l}^{2}+2 e B \nu}+\Delta_{l}}\right|\right] \\
& +\frac{1}{4 \pi^{2}} \sum_{\nu=1}^{\nu_{\max }}\left[k_{f, \nu,-1}^{(l)} E_{f}^{(l)}+\left(\sqrt{m_{l}^{2}+2 e B \nu}-\Delta_{l}\right)^{2} \ln \left|\frac{k_{f, \nu,-1}^{(l)}+E_{f}^{(l)}}{\sqrt{m_{l}^{2}+2 e B \nu}-\Delta_{l}}\right|\right]
\end{aligned}
$$

The total energy density of the n-p-e- $\mu$ system is[11]

$$
\varepsilon=\varepsilon_{p}+\varepsilon_{n}+\varepsilon_{e}+\varepsilon_{\mu}+\frac{1}{2} m_{\sigma}^{2} \sigma^{2}+U(\sigma)+\frac{1}{2} m_{\delta}^{2} \delta_{0}^{2}+\frac{1}{2} m_{\omega}^{2} \omega_{0}^{2}+\frac{1}{2} m_{\rho}^{2} R_{0,0}^{2}+\frac{B^{2}}{8 \pi} .
$$

where the last term is the contribution from the external magnetic field.

Because of the charge neutrality and chemical equilibrium the pressure of the system 
can be obtained by

$$
P=\sum_{i} \mu_{i} \rho_{i}-\varepsilon=\mu_{n} \rho_{b}-\varepsilon
$$

\section{$3 \quad$ Numerical results}

From above expressions, one can obtain the nucleon effective masses and the EOS of the system after solving the meson field equation, of (4)-(7), numerically. The chemical equilibrium conditions of $\mu_{n}=\mu_{p}+\mu_{e}$ and $\mu_{e}=\mu_{\mu}$, as well as the charge neutrality $\rho_{p}=$ $\rho_{e}+\rho_{\mu}$ are applied in the iteration procedure. The nucleon-meson coupling constants and the coefficients in the scalar field self-interactions are obtained by adjusting to the bulk properties of symmetric nuclear matter where the magnetic field is absence. Furthermore, for symmetric nuclear matter the leptons are omitted and protons and neutrons have equal densities. The saturation properties consist of the binding energy $(E / A)$, compression $\operatorname{modulus}(K)$, symmetry $\operatorname{energy}\left(a_{\text {sym }}\right)$, the effective mass $\left(m_{N}^{*} / M_{N}\right)$ and the pressure $p$. The binding energy can be obtained by $E / A=\varepsilon / \rho_{b}-M_{b}$. The symmetry energy reads[5]

$$
a_{\text {sym }}=\frac{1}{2} C_{\rho}^{2} \rho_{0}+\frac{k_{f}^{2}}{6 \sqrt{k_{f}^{2}+m^{* 2}}}-C_{\delta}^{2} \frac{m^{* 2} \rho_{0}}{2\left(k_{f}^{2}+m^{* 2}\right)\left(1+C_{\delta}^{2} A\left(k_{f}, m^{*}\right)\right)}
$$

where

$$
A\left(k_{f}, m^{*}\right)=\frac{4}{(2 \pi)^{3}} \int_{0}^{k_{f}} \frac{k^{2} d^{3} k}{\left(k^{2}+m^{* 2}\right)^{3 / 2}}
$$

is a function of the Fermi momentum, $k_{f}=k_{f}^{(p)}=k_{f}^{(n)}$, and the effective mass, $m^{*}=$ $m_{p}^{*}=m_{n}^{*}$. For symmetric nuclear matter at saturation density $\rho_{0}$, we have defined $C_{\sigma}=g_{\sigma} / m_{\sigma}, C_{\omega}=g_{\omega} / m_{\omega}, C_{\rho}=g_{\rho} / m_{\rho}, C_{\delta}=g_{\delta} / m_{\delta}$. In the presence of $\delta$-fields, the compression modulus is calculated to be

$$
\begin{aligned}
\frac{1}{9} K & =\left.k_{f}^{2} \frac{\partial^{2}}{\partial k_{f}^{2}}\left(\frac{\varepsilon_{0}}{\rho}\right)\right|_{\rho=\rho_{0}} \\
& =C_{\omega}^{2} \rho_{0}+\sum_{N} \frac{k_{f}^{2}}{6 E_{f}^{(N)}}+\frac{\rho_{0}}{2}-\frac{\left(B_{p}+B_{n}\right)^{2}+C_{\delta}^{2}\left[f_{\sigma}\left(B_{p}-B_{n}\right)^{2}+2 A_{n} B_{p}^{2}+2 A_{p} B_{n}^{2}\right]}{2 f_{\sigma}+C_{\delta}^{2}\left[\left(A_{n}+A_{p}\right) f_{\sigma}+2 A_{n} A_{p}\right]+A_{n}+A_{p}},
\end{aligned}
$$


where $\mathrm{N}=\mathrm{n}, \mathrm{p}$, and

$$
\begin{aligned}
& A_{N}=\frac{6 \rho_{s}^{N}}{m_{N}^{*}}-\frac{3 \rho_{0}}{E_{N}} \\
& B_{N}=\frac{m_{N}^{*}}{E_{N}}, \quad f_{\sigma}=\frac{U(\sigma)}{g_{\sigma}^{2}} .
\end{aligned}
$$

Recently, improved empirical data for the compressibility and symmetry energy are available [19, 20, 21], which benefit to the study of evident isospin asymmetric nuclear matter. In this work we adopt the parameter sets obtained in Ref.[4]. The coupling constants and the corresponding saturation properties of nuclear matter are listed in Table 1 . One can see that SetA with and without the $\delta$-field produce the same saturation properties, which fits well into the range of new empirical data. Therefore, it is suitable to be used to investigate the $\delta$-field effect in isospin asymmetric matter. Another set of parameters GM3[22] is widely used in neutron-star matter calculations. The results for neutron-star matter without magnetic fields are presented in Fig.1.

The dotted lines in Fig. 1(a) denote the effective masses of neutrons and protons as functions of baryon densities in a n-p-e- $\mu$ system reckoned in the model of SetA including the $\delta$-field. The splitting of proton and neutron masses can affect the transport properties of dense matter[5]. The difference between the proton and the neutron effective mass inclines to decrease with the increasing of the baryon density. It indicates that the strength of $\delta$-field $-g_{\delta} \delta$ decreases with densities. For comparison the results for the model of SetA without $\delta$-field and set GM3 are presented in the figure too. In Fig. 1(b) and Fig. 1(c) we show the pressure densities and energy densities as functions of the baryon density. The deviation between the results of $\operatorname{Set} \mathrm{A}(N L \sigma \omega \rho)$ and $\operatorname{Set} \mathrm{A}(N L \sigma \omega \rho \delta)$ are negligible. Although the proton fraction increases by including the $\delta$-field[4], the EOS has little change due to the fact that the energy density and pressure density display the average contributions of protons and neutrons, and the effects of isospin vector fields are small and cancel somewhat. Alternatively, because of a larger effective mass the results of Set GM3 deviate from SetA evidently. In the following calculations, we present the numerical results under strong magnetic fields by separating the cases with and without the inclusion 
of the AMM effects.

\subsection{Effect of the magnetic fields without AMM terms}

We consider magnetic fields in the range of $10^{12}-10^{20} \mathrm{G}$. There are two characteristic strengths of magnetic fields for the problem involved, which are critical fields for electrons $\left(B_{c}^{e}=4.414 \times 10^{13} \mathrm{G}\right)$ and $\operatorname{protons}\left(B_{c}^{p}=1.487 \times 10^{20} \mathrm{G}\right)[11]$. We are more interested in the results at the vicinity of $B=B_{c}^{e}$ and ultrastrong magnetic fields around $B=10^{18} \mathrm{G}$. Therefore, in the following calculations we consider magnetic fields of $B=10^{12} \mathrm{G}, 10^{13} \mathrm{G}, 10^{15} \mathrm{G}, 10^{5} \times B_{c}^{e}, 10^{19} \mathrm{G}, 3 \times 10^{19} \mathrm{G}$. For comparison, the results for $B=0$ will be presented too. In order to manifest the influence of magnetic fields on neutron-star matter, in the results of pressure and energy density given below the magnetic energy part will be excluded.

Figure 2 depicts the nucleon effective masses as functions of baryon densities for various magnetic fields. From Fig. 2(a) it can be seen that the effective mass of protons at $B=10^{12} \mathrm{G}, 10^{13} \mathrm{G}$ is much larger than that without magnetic field. The results of $B=10^{15} \mathrm{G}, 10^{5} \times B_{c}^{e}, 10^{19} \mathrm{G}$ are indistinguishable from those of $B=0 \mathrm{G}$. When the field strength further increases, a smaller $m_{p}^{*}$ was obtained. The enhancement and suppression of proton effective masses mainly results from the variation of proton fraction caused by the effect of magnetic fields. The effective masses of neutrons given in Fig. 2(b), however, have little changes. The different response of the proton and neutron effective mass can be explained in Figure 3, where the meson field strength as functions of baryon densities are displayed. Fig. 3(a) gives the $\sigma$-field strength $-g_{\sigma} \sigma$ as a function of the density. One can find that the curves behave similar to that of the proton effective mass, except that the $m_{p}^{*}$ varies more rapidly due to the including of the $\delta$-field. Fig. 3(b) delineates that the $\delta$ field strength changes substantially with magnetic fields around $B=10^{13} \mathrm{G}$. The nucleon effective masses are entirety defined by the $\sigma$ - and $\delta$-field. The proton effective mass is enhanced significantly at $B=10^{12} \mathrm{G}$ and $B=10^{13} \mathrm{G}$ because of the large cancellation between the $\sigma$ - and $\delta$-field. With the increasing of the magnetic field, the strength of the $\sigma$ - 
and $\delta$-field approaches the field free case, so does the proton effective mass. The situation for neutron effective masses is different. The including of the $\delta$-field lowers the effect of magnetic fields on the $\sigma$-field. Thus the neutron effective mass is almost independent with magnetic fields. One can also see that at larger magnetic field $\left(B>10^{15} \mathrm{G}\right)$ and higher density the $\delta$-field strength is near zero. That indicates that the effective masses of neutrons and protons tend to be same at higher density. At ultrastrong magnetic field $\left(B=3 \times 10^{19} \mathrm{G}\right)$, the $\delta$-field strength turns out to be negative at density of $\rho>2 \rho_{0}$. We also present the strength of $\omega$-field $\left(g_{\omega} \omega_{0}\right)$ and $\rho$-field $\left(g_{\rho} R_{0,0}\right)$ in Fig. 3(c) and Fig. $3(\mathrm{~d})$, respectively. The $\omega$-field strength is solely defined by the baryon density and thus linear with it. The $\rho$-field represents the isospin asymmetry of nuclear matter. From Fig. $3(\mathrm{~d})$, one can find that the $\rho$-field strength for magnetic fields of $B=10^{12} \mathrm{G}$ and $10^{13} \mathrm{G}$ are much larger than that for others, which means that the proton fractions are very small and the neutron-star matter are extremely asymmetric. At ultrastrong magnetic fields ( $B=3 \times 10^{19}$ ) the $\rho$-field is almost zero, i.e, the density of protons is approximately equal to that of neutrons. The neutron-star matter inclines to isospin symmetric at very large magnetic field.

Figure 4 shows the EOS of neutron-star matter under magnetic fields for SetA(NL $\sigma \omega \rho \delta)$ with the AMM terms excluded. For pressure and energy density we present the matter part only. In Fig. 4(a), the energy per nucleon as a function of the baryon density is depicted for various magnetic fields., while the pressure is given in Fig. 4(b) and (c). One can see that the equation of state becomes stiffer around $B=10^{12} \mathrm{G}$ compared to the field-free case. At the range of $B=10^{15} \mathrm{G}-10^{19} \mathrm{G}$ the EOS is indistinguishable to that of $B=0$. At even larger magnetic field the EOS comes out to be softer which is in accordance with previous studies[11]. The variation of EOS with magnetic field strength can be understood by the particle fraction as discussed before. The results of $\operatorname{Set} \mathrm{A}(N L \sigma \omega \rho)$ without $\delta$-field are shown in Figure 5. The general trends of the nucleon effective mass, the $\rho$-field and EOS are similar to that as depicted in Fig. 2 and Fig. 4, but the magnitude of variation is suppressed. Thus one may conclude that the magnetic field effects 
change the proton fraction and the $\delta$-field enhances the isospin asymmetric effects.

From above, we can find that the $\delta$-field leads to splitting of nucleon effective masses. Under magnetic field the proton effective mass decrease rapidly with magnetic field and in range of $10^{15} G<B<10^{19} \mathrm{G}$ the results is almost indistinguishable from that of $B=0$. The neutron effective mass have little change for different fields because the effects of magnetic field on $\sigma$-field and $\delta$-field counteract. The EOS change a lot for different magnetic field because of the change of proton fraction. By including of $\delta$-field, the EOS of neutron-star matter is stiffer for strong magnetic fields $\left(B<10^{15} \mathrm{G}\right)$ and become softer for ultrastrong magnetic fields $\left(B>10^{18} \mathrm{G}\right)$. The effect of $\delta$-field decrease with magnetic field and is little at magnetic field of $B>10^{18} \mathrm{G}$. The EOS for $10^{15}<B<10^{19} \mathrm{G}$ is similar with that of $B=0$ and at ultrastrong magnetic field $\left(B>10^{19} \mathrm{G}\right)$ the neutron-star matter tends to symmetric. From above analysis, we also find that the fraction of proton play an important role in the description of nucleon effective mass and the EOS of neutron-star matter.

\section{$3.2 \quad$ Effect of AMM terms}

In previous works[11], the effect of AMM terms of nucleons on the EOS of $n-p-e-\mu$ system in the absence of $\delta$-field are studied in detail. In our studies, we will introduce the AMM term of muons. At the magnetic field $B=10^{17}-10^{20} \mathrm{G}$, the densities of muons are comparable with baryon densities[11]. The effect of the muon AMM term is then expected to make sense. We adopt the value $a_{\mu}=1165203(8) \times 10^{-10}(0.7 p p m)[17,18]$, which is a present word average experimental value. In the following we show the results including the AMM terms of nucleons and muons, while the effect of the electron AMM term will be investigated in the next section.

The proton effective mass as a function of the baryon density is shown in Fig. 6(a). One can find that the results for $B<10^{19} \mathrm{G}$ have very little change compared with the case without AMM terms. For $B=3 \times 10^{19} \mathrm{G}$ the proton effective mass no more decreases monotonically but tends to reach certain situation at high density. A similar 
situation is exhibited for the neutron effective mass as shown in Fig. 6(b). AT ultra high magnetic field the $m_{n}^{*}$ becomes larger than the field-free case while for lower B they are indistinguishable. This is mainly caused by the effects of AMM terms on the $\sigma$-field. In Figure 7 one can see that the scalar field is increased at large field, especially for high densities. The changes on the $\delta$ - and $\rho$-field are negligible for with and without AMM terms.

The EOS of the system are shown in Figure 9. Again, the main modifications due to the including of AMM terms come out for high fields of $B>10^{19} \mathrm{G}$. The energy per nucleon becomes strongly binding at lower densities. Alternately, a stiffer pressure is displayed compared to the case without AMM terms. It should be mentionable that the EOS is only for matter, the magnetic energy itself has not yet been added.

We can conclude from above that at ultrastrong magnetic fields $\left(B>10^{19} \mathrm{G}\right)$ the proton effective masse and neutron effective mass all become larger by including of AMM terms. And the effective masse of neutron is bigger than that of proton effective masses at lower densities $\left(\rho<4 \rho_{0}\right)$, since in this regions the density of protons is larger than that of neutrons. The EOS becomes much stiffer at $B=10^{19} \mathrm{G}$ because of the effect of AMM terms. The nucleon effective mass and EOS of this system have no evident change under magnetic fields of $B<10^{15} \mathrm{G}$. The AMM terms play an important role only under very strong magnetic field of $B>10^{18} \mathrm{G}$. and the effect of AMM terms decrease with magnetic field. On the other hand, the effect of $\delta$-field decreases with magnetic field and is very small at $B>10^{18} \mathrm{G}$. Its main contribution was shown at $B \sim 10^{12} G$

\subsection{Results including AMM of electrons}

The critical field of electrons is about $10^{13} \mathrm{G}$. Most of magnetic field strengths considered in this work are around or well beyond this point. It is generally believed that the high-order terms stemming from the vacuum polarization of electrons in an external magnetic field [23] may get into work near the critical point and cancel the electron AMM term. Nevertheless, it is interesting to check the effects of the electron AMM term nu- 
merically in the present system. The results including the $\delta$-field and AMM terms of all relevant particles are depicted in Figure 9. Fig. 9(a) and (b) show the nucleon effective masses as functions of baryon densities. The pressure as functions of baryon densities and energy densities are show in Fig. 10(c). One can see that the nucleon effective masses and EOS have negligible changes compared with the situation excluding the AMM of electrons.

\section{Summary and outlook}

We have studied the properties of the neutron-star matter consisting of n-p-e- $\mu$ in strong magnetic fields. For nuclear interactions we applied the relativistic mean field theory with the exchange of $\sigma_{-}^{-}, \omega-, \rho$ - and $\delta$-mesons. Our main interest is to investigate the influences of isospin vector field on the asymmetric matter in the presence of magnetic fields. The effects of AMM terms of nucleons and leptons are included. Two sets of coupling constants with and without $\delta$-field are used in calculations. The nucleon effective masses and EOS are studied in detail. Interesting results have been found for two regions of magnetic field strength. At lower field of $B \sim 10^{12} \mathrm{G}$, where the AMM terms play no role, the proton effective mass was enhanced significantly compared to the case of $B=0$. The equation of state becomes much stiffer. This is mainly caused by the change of proton fraction. The neutron effective mass is almost independent of magnetic fields because the effect of $\sigma$ - and $\delta$-field cancel to some extent. In the range of $B 10^{15}--10^{18} \mathrm{G}$, no obvious differences were found both on the nucleon effective masses and EOS for with and without the magnetic field. At larger field of $B \sim 10^{19} \mathrm{G}$, the proton effective mass increases with magnetic field at high density $\left(\rho>5 \rho_{0}\right)$ while at lower density it becomes less than the neutron effective mass. The EOS of neutron-star matter is softer at ultrastrong field but becomes stiffer with the inclusion of AMM terms. Besides, one can find that the neutronstar matter tends to be symmetric at the range calculated. Compared with the results without $\delta$-field, we find that the effect of $\delta$-field decreases with magnetic field and becomes little at $B>10^{19} \mathrm{G}$. It can also be found that the effect of AMM terms increases with 
magnetic field and is very little when the field strength $B<10^{15} \mathrm{G}$. At the end, we have presented the results including $\delta$-field and AMM of nucleons, muons and electrons, and find that the effect of the electron AMM is very little. Particularly, the proton fraction can be proved to play an important role in descriptions of properties of neutron-star matter .

The vector self-interaction terms of $\omega$-field can influence the maximum mass, the rotational frequency and cooling properties of neutron stars[24]. The spin polarization of protons probably affect the structure and composition of neutron stars. These questions will be studied in forthcoming work. With the densities increasing, the hyperon and quark degrees of freedom must be considered for the core of neutron stars [3, 24]. The interactions of quarks are very deferent from that of nucleons and can lead to many new results[25]. All these can influence the EOS of neutron star matter and warrant further studies.

Acknowledgements: The authors are grateful to N. Van. Giai, J. Schaffner and B. Liu for fruitful discussions. This work was supported by the National Natural Science Foundation of China under Grant No. 10275072. 


\section{References}

[1] J. D. Walecka, Ann. Phys(N.Y). 83, 491(1974).

[2] B. D. Serot and J. D. Walecka, Adv. Nucl. Phys. 16, 1(1986).

[3] J. Schaffner and I. N. Mishustin, Phys. Rev. C53, 1416(1996).

[4] B. Liu, H. Guo, V. Baran, M. Di. Toro and V. Greco, nucl-th/0409014, B. Liu, V. Greco, V. Baran, M. Colonna, and M. Di. Toro, Phys. Rev. C65, 045201(2002)

[5] S. Kubis and M. Kutschera, Phys. Lett. B399, 191(1997).

[6] D. P. Menezes and C. Providencia, Phys. Rev. C70, 058801(2004).

[7] Bao-An Li, phys. Rev. C69, 064602(2004).

[8] Bao-An Li and Lie-Wen Chen, nucl-th/0508024.

[9] A. Melatos, APJ. L77, 519(1999).

[10] I. Lerche and D. N. Schramm, APJ. 216, 881(1977).

[11] A. Broderick, M. Prakash and J. M. Lattmer, APJ. 537, 351(2000).

[12] C. Y. Cardall, M. Prakash and J. M. Lattimer, APJ. 554, 322(2001).

[13] S. Chakrabarty, D. Bandyopadhyay, and S. Pal, Phys. Rev. Lett. 78, 2898(1997)

[14] N. K. Glendenning, APJ. 293, 470(1985).

[15] J. Boguta, A.R. Bodmer, Nucl.Phys. A292, 413(1977).

[16] G. Mao, N. V. Kondratyev, A. Iwamoto, Z. Li, X. Wu, W. Greiner, N. I. Mikhailov, Chin. Phys. Lett. 20, 1238(2003).

[17] G. W. Bennett, B. Bousquet et al., Phys. Rev. Lett. 89, 101804(2002). 
[18] Mark Byrne, Christopher Kolda and Jason E. Lennon, Phys. Rev. D67, $075004(2003)$.

[19] D. Vretenar, T. Niksic and P. Ring, Phys. Rev. C68, 024310(2003).

[20] G. Colo, N. Van Giai, J. Meyer, K. Bennaceur and P. Bonche, Phys. Rev. C70, 024307(2004).

[21] V. B. Soubbotin, V. I. Tselyaev, X. Vinas, Phys. Rev. C69, 064312(2004)

[22] N. K. Glendenning and S. A. Moszkowski, Phys. Rev. Lett. 67, 2414(1991).

[23] R.C. Duncan, astro-ph/0002442.

[24] N. K. Glendenning, Z. Phy. 326, 57(1987).

[25] P. Wang, S. Lawley, D. B. Leinweber, A. W. Thomas, A. G. Williams, nuclth/0506014 
TABLE 1

Parameter sets and the corresponding saturation properties of nuclear matter

\begin{tabular}{|c|c|c|c|c|c|c|c|c|c|c|c|}
\hline Parameter Sets & $\begin{array}{c}C_{\sigma}^{2} \\
(\mathrm{fm})\end{array}$ & $\begin{array}{c}C_{\omega}^{2} \\
(\mathrm{fm})\end{array}$ & $\begin{array}{c}C_{\rho}^{2} \\
(\mathrm{fm})\end{array}$ & $\begin{array}{c}C_{\delta}^{2} \\
(\mathrm{fm})\end{array}$ & $\begin{array}{c}\mathrm{b} \\
\left(f m^{-1}\right)\end{array}$ & $\mathrm{c}$ & $\begin{array}{c}\rho_{0} \\
f m^{-3}\end{array}$ & $\begin{array}{c}\mathrm{E} / \mathrm{A} \\
(\mathrm{MeV})\end{array}$ & $m_{N}^{*} / M_{N}$ & $\begin{array}{c}a_{\text {sym }} \\
(\mathrm{MeV})\end{array}$ & $\begin{array}{c}\mathrm{K} \\
(\mathrm{MeV})\end{array}$ \\
\hline $\operatorname{set} A(N L \rho)$ & 10.32924 & 5.42341 & 0.94999 & 0.0000 & 0.03302 & -0.00483 & 0.16 & -16.0 & 0.75 & 31.3 & 240 \\
\hline $\operatorname{Set} \mathrm{A}(\mathrm{NL} \rho \delta)$ & 10.32924 & 5.42341 & 3.1500 & 2.5000 & 0.03302 & -0.00483 & 0.16 & -16.0 & 0.75 & 31.3 & 240 \\
\hline GM3 & 9.927 & 4.820 & 1.198 & 0.000 & 0.041205 & -0.002421 & 0.153 & -16.3 & 0.78 & 32.5 & 240 \\
\hline
\end{tabular}



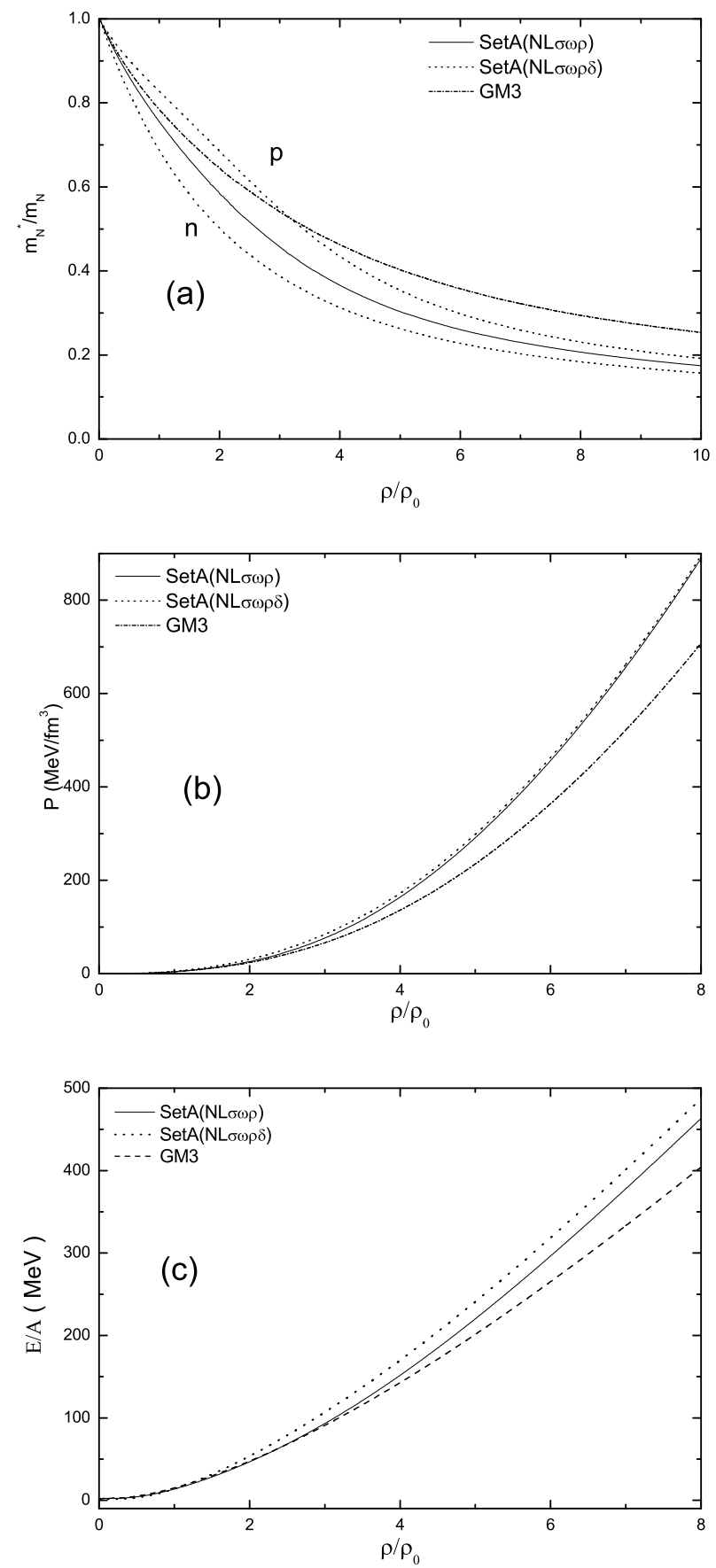

Figure 1: Nucleon effective masses $m_{N}^{*} / M_{N}(\mathrm{a})$ and pressure(b) as functions of baryon densities $\rho / \rho_{0}$ for the neutron-star matter without magnetic fields; (c) shows the energy per nucleon as a function of baryon density. 

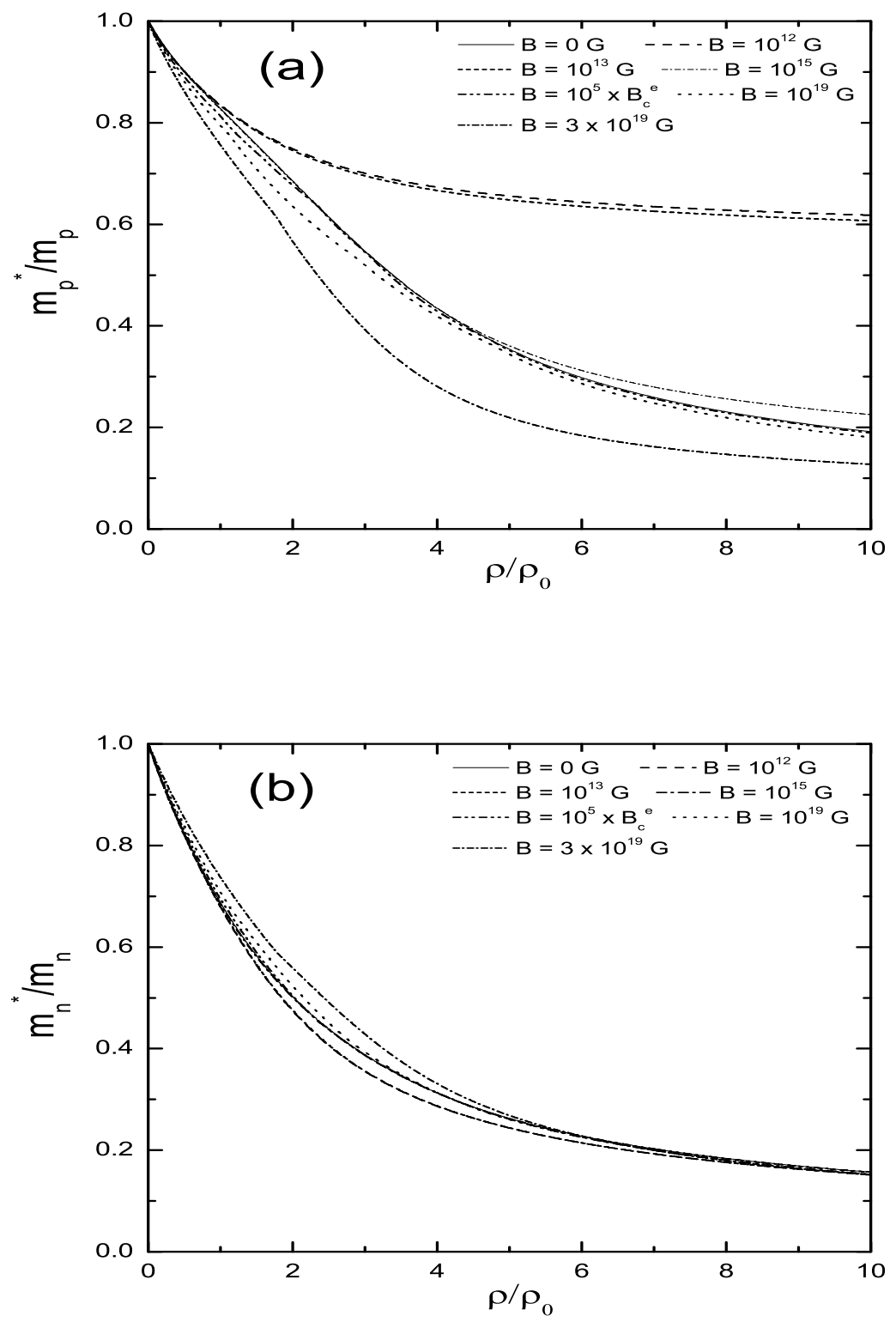

Figure 2: Effective masses of protons(a) and neutrons(b) as functions of the density for different magnetic field strengths. The parameter $\operatorname{Set} \mathrm{A}(N L \sigma \omega \rho \delta)$ has been used in calculations. 

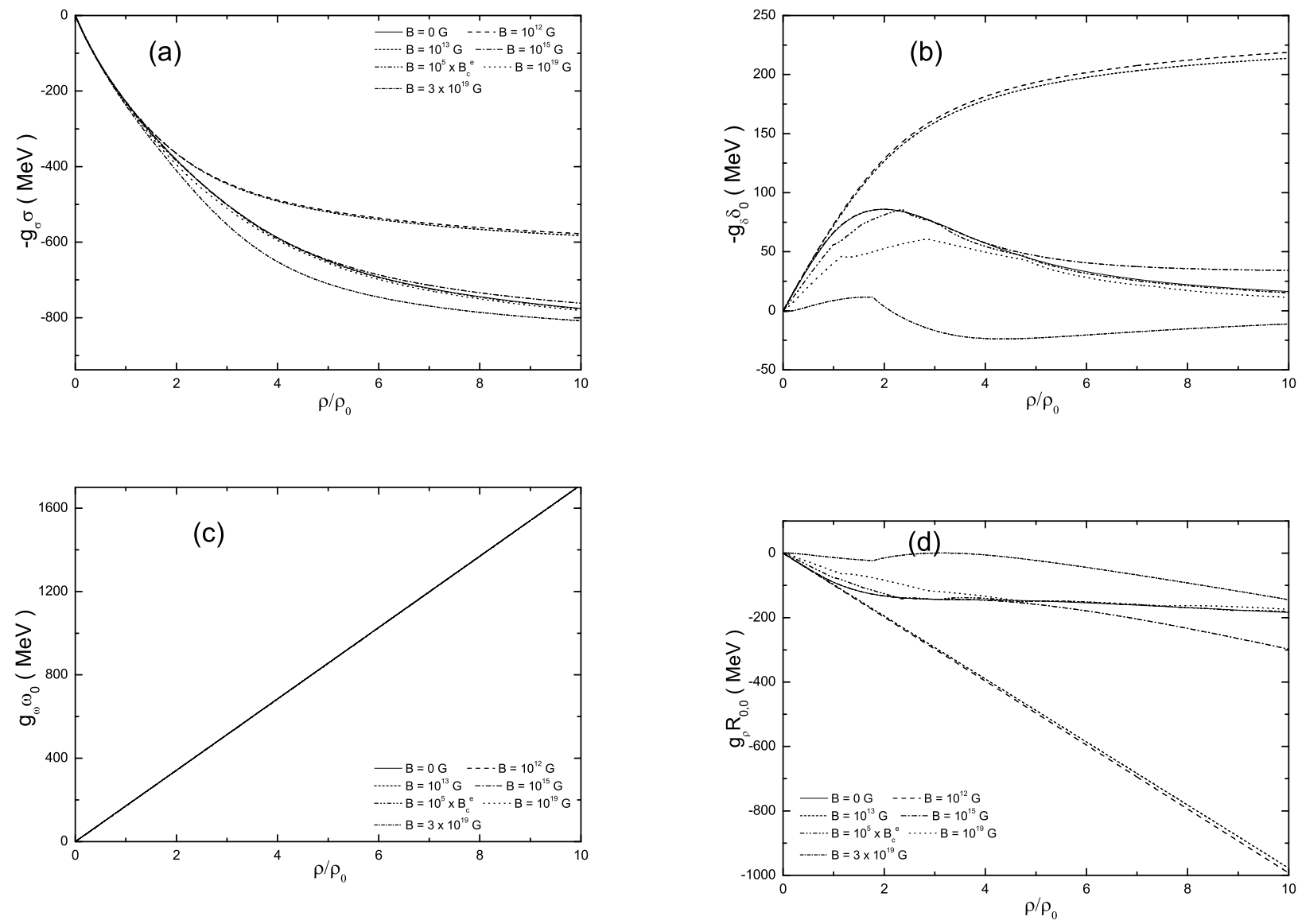

Figure 3: The strength of the $\sigma$-field(a), $\delta$-field(b), $\omega$-field(c) and $\rho$-field(d) as functions of densities for different magnetic fields. 

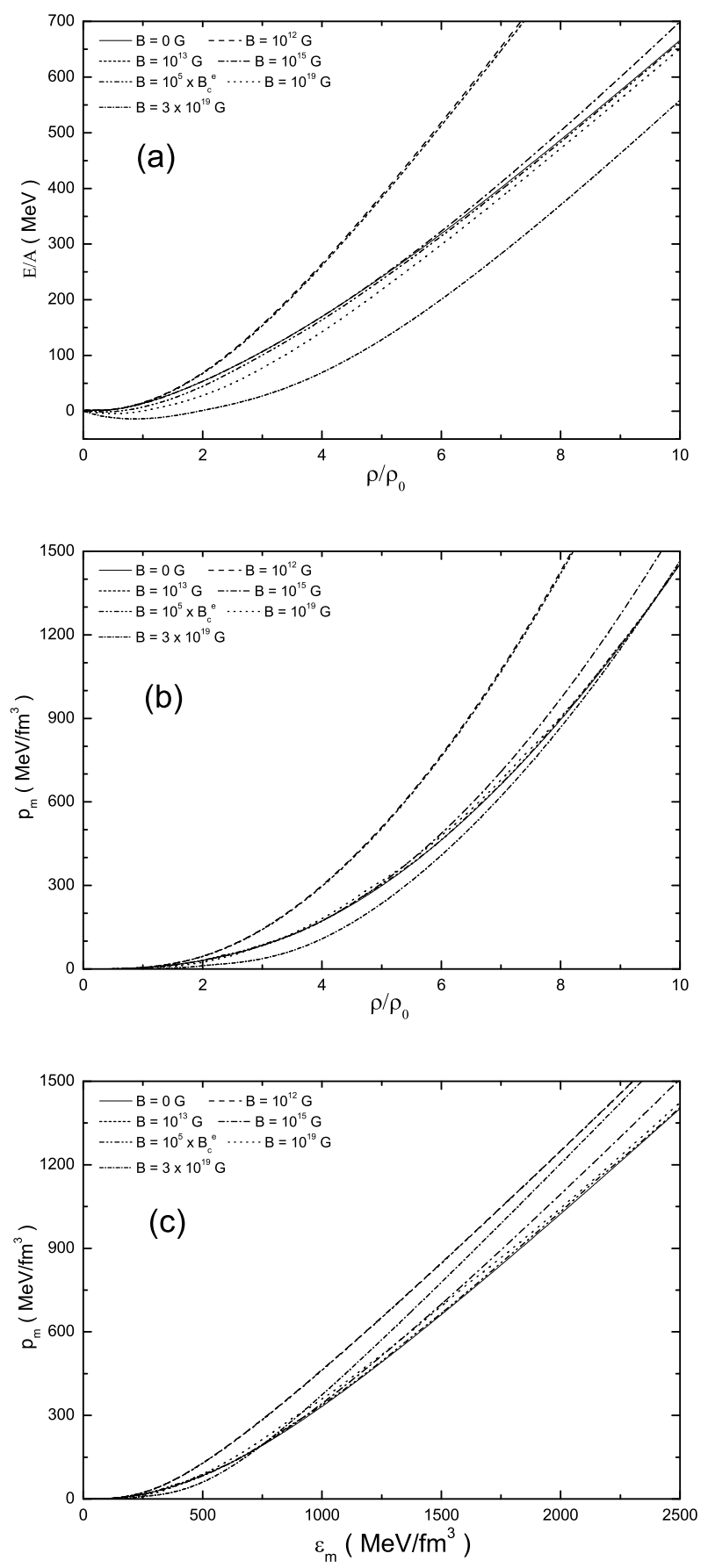

Figure 4: Energy per nucleon(a) and the matter part of pressure density $p_{m}(\mathrm{~b})$ as functions of the baryon density, Figure(c) shows the $p_{m}$ as a function of matter energy densities $\varepsilon_{m}$. 

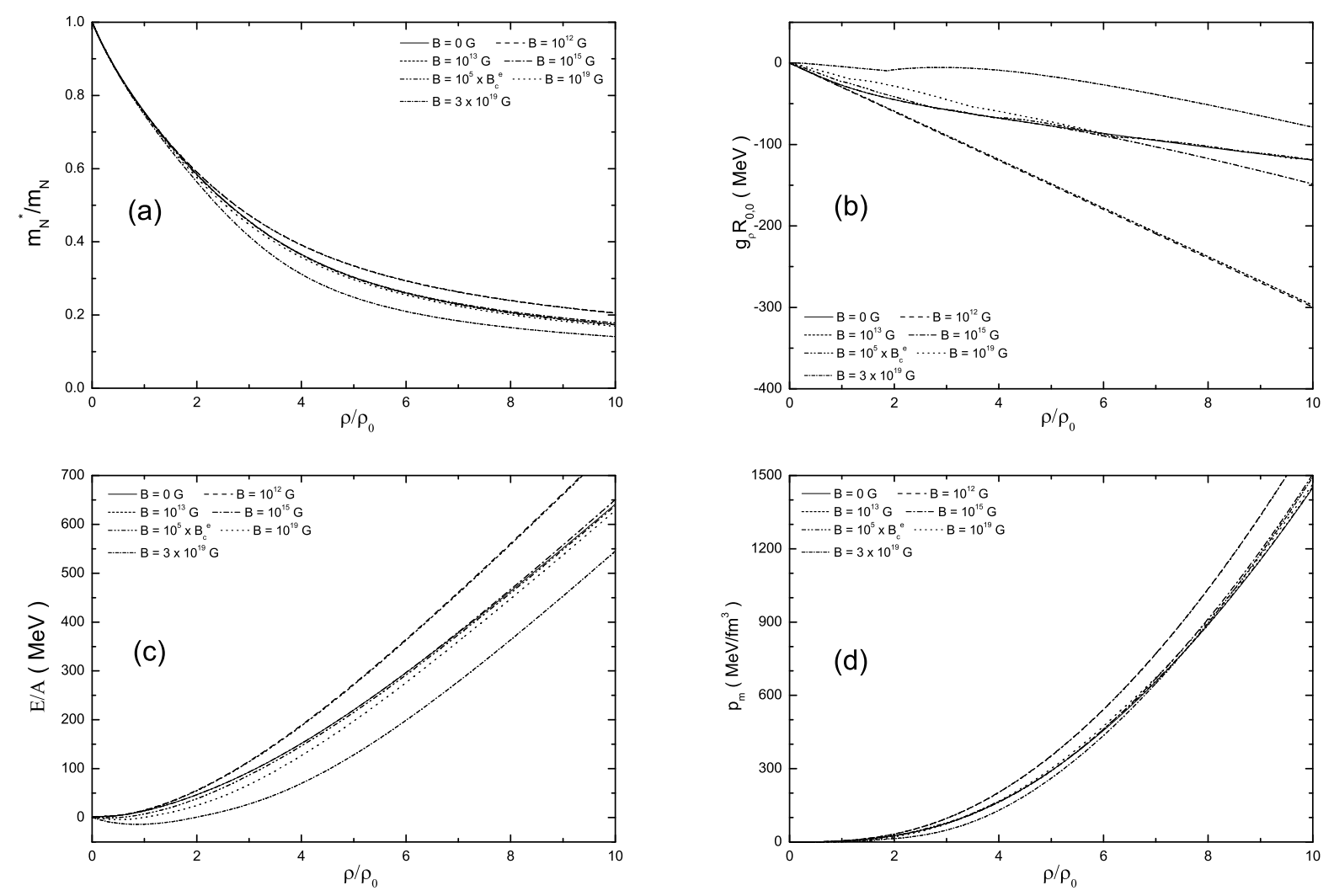

Figure 5: The results for n-p-e- $\mu$ system calculated with $\operatorname{SetA}(N L \sigma \omega \rho)$ without $\delta$-field. (a) shows the nucleon effective mass as a function of the baryon density for magnetic fields as presented in Figure2; (b) displays the $\rho$-field strength $g_{\rho} R_{0,0} ;(\mathrm{c})$ and (d) show the energy per nucleon and pressure density, respectively. 

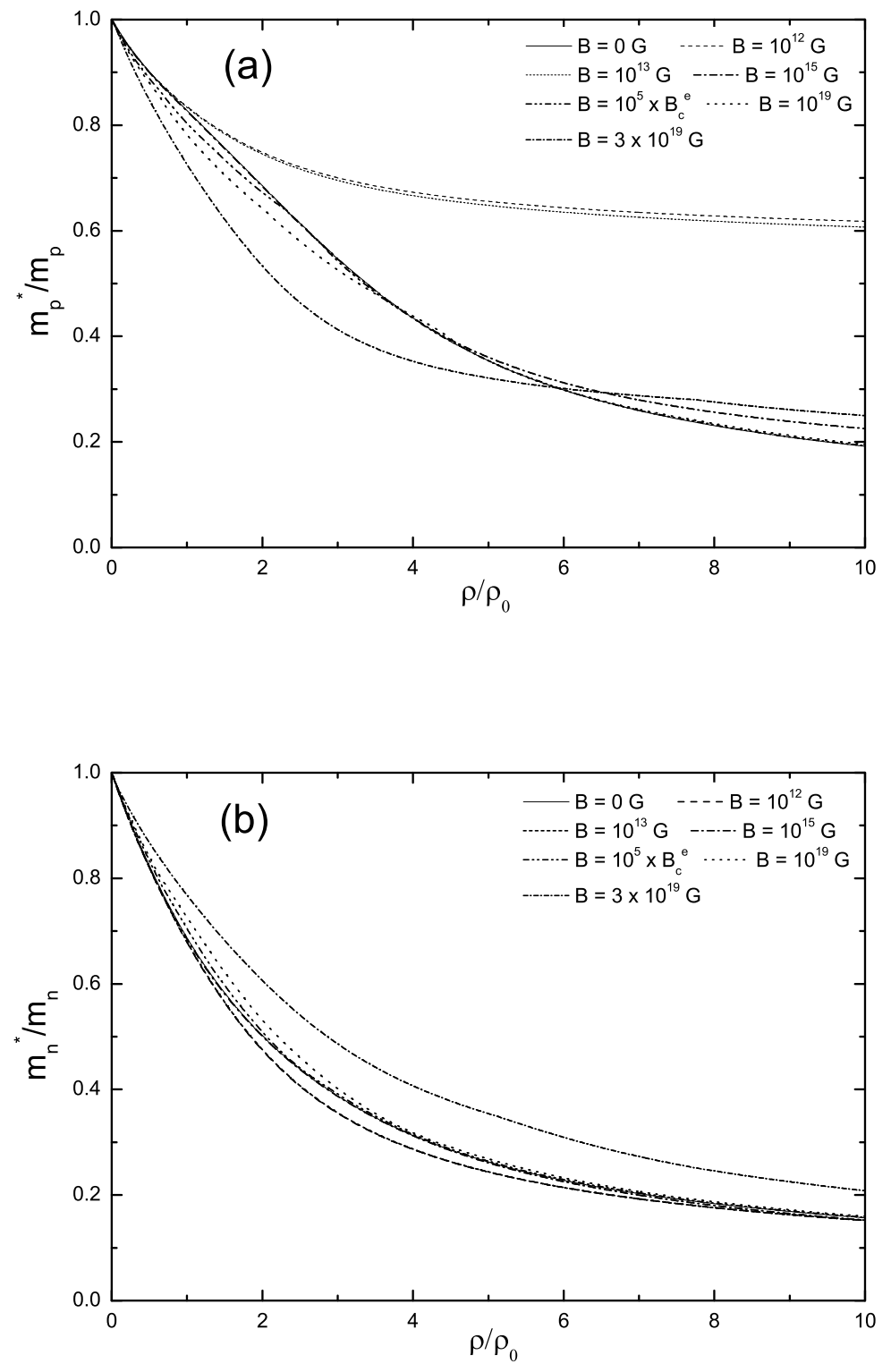

Figure 6: Same as Figure 2, except that the AMM terms of nucleons and muons are included. 

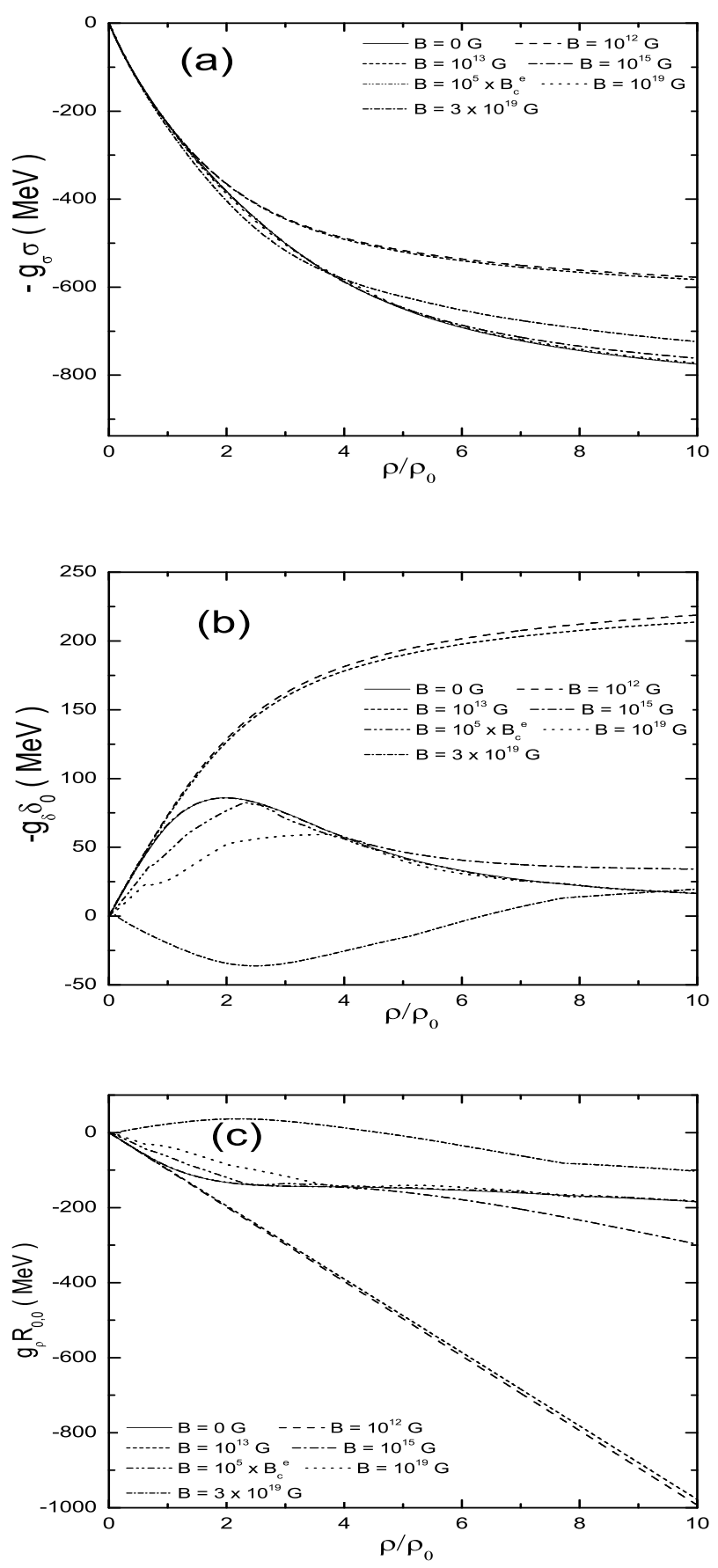

Figure 7: Same as Figure 3, except that the AMM terms of nucleons and muons are included. 

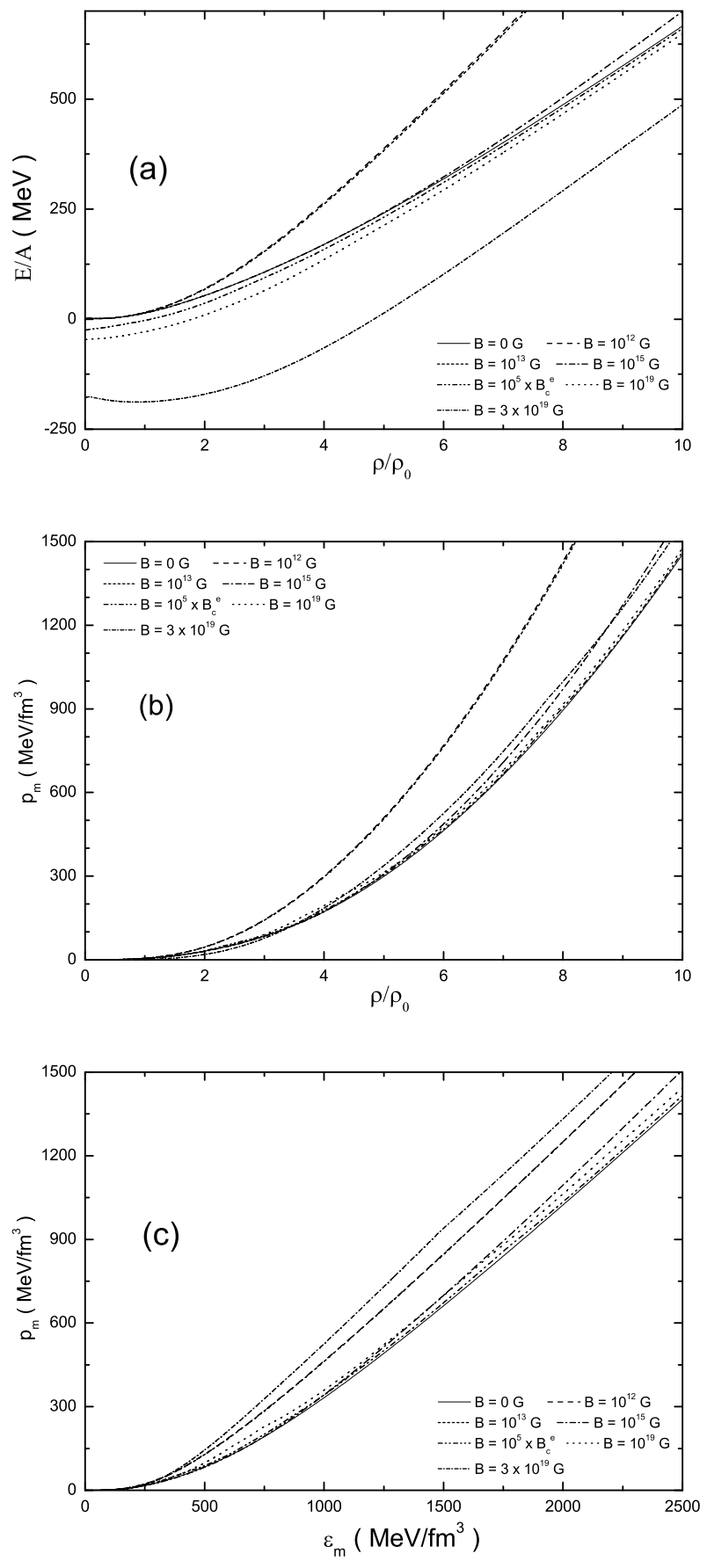

Figure 8: Same as Figure 4, except that the AMM of nucleons and muons are included. 

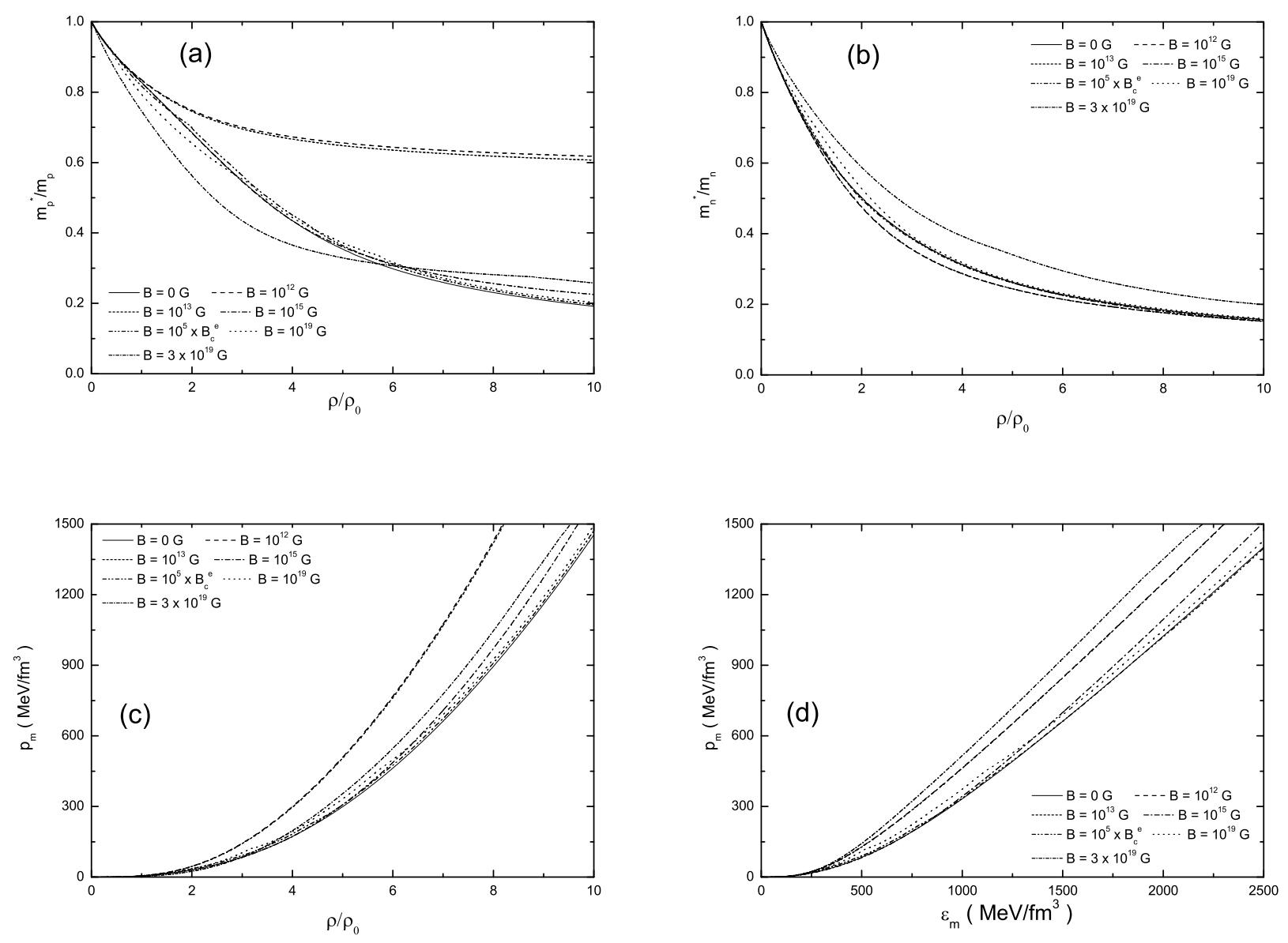

Figure 9: Results including the effect of AMM of electron for model SetA(NL $\sigma \omega \rho \delta)$. (a) and (b) present the effective masses of proton and neutron, respectively; (c) and (d) show the pressure densities as functions of baryon densities and energy densities, respectively. 